\title{
Uterine Sarcoma: Clinical Presentation, Treatment and Survival Outcomes in Thailand
}

\author{
Chalermrat Potikul, Siriwan Tangjitgamol*, Jakkapan Khunnarong, Sunamchok \\ Srijaipracharoen, Thaovalai Thavaramara, Kamol Pataradool
}

\begin{abstract}
Background: Uterine sarcoma is a group of rare gynecologic tumors with various natures, and different lines of treatment. Most have a poor treatment outcome. This study targeted clinical characteristics, treatment, overall survival (OS), progression-free survival (PFS), and prognostic factors in uterine sarcoma patients in one tertiary center for cancer care. Materials and Methods: Uterine sarcoma patients who were treated at the Department of Obstetrics and Gynecology, Faculty of Medicine Vajira Hospital between January 1994 and December 2014 were identified. Clinico-pathological data were analyzed. Prognostic outcomes were examined by Kaplan-Meier curves and Cox regression analysis. Results: We identified 46 uterine sarcoma patients: 25 carcinosarcoma (CS) $(54.3 \%)$, 15 leiomyosarcoma (LMS) $(32.6 \%)$, and 6 undifferentiated uterine sarcoma (UUS) (13.1\%) cases. Mean age was 54.0 \pm 11.9 years (range 25-82 years). Abnormal uterine bleeding was the most common presenting symptom (63.0\%). Among 33 patients $(71.7 \%)$ who had pre-operative tissue collected, diagnosis of malignancy was correct in $29(87.9 \%)$. All patients received primary surgery and retroperitoneal lymph nodes were resected in $34 \mathbf{( 7 3 . 9 \% )}$. After surgery, $5(\mathbf{1 0 . 9 \%})$ had gross residual tumors. Stage I disease was most commonly found $\mathbf{( 5 6 . 5 \% )}$. Adjuvant treatment was given to $27(58.7 \%)$, most commonly chemotherapy. After a median follow-up of 16.0 months (range 0.8-187.4 months), recurrence was encountered in 22 patients $(47.8 \%)$. Median time to recurrence was 5.8 months (range1.0-105.5 months). Distant metastasis was more common than local or locoregional failure. The 2-year PFS was $45.2 \%$ (95\% confidence interval [CI], 30.6\%-59.7\%) and the 2-year OS was $48.3 \%(95 \% \mathrm{CI}, 33.3 \%-60.7 \%)$. Multivariable analyses found residual disease after surgery as a significant factor only for PFS. Conclusions: Uterine sarcoma is a rare tumor entity. Even with multimodalities of treatment, the prognosis is still poor. Successful cytoreductive surgery is a key factor for a good survival outcome.
\end{abstract}

Keywords: Uterine sarcoma - clinical characteristics - survival - prognostic factors - Thailand

Asian Pac J Cancer Prev, 17 (4), 1759-1767

\section{Introduction}

Uterine sarcoma is a rare tumor entity, accounting for $4-9 \%$ of all uterine malignancies (Brooks et al., 2004). Histological types of pure uterine sarcoma are leiomyosarcoma (LMS), endometrial stromal sarcoma (ESS), and undifferentiated uterine sarcoma (UUS) while mixed sarcoma and epithelial tumors are carcinosarcoma (CS) and adenosarcoma (AS) (Oliva et al., 2014; Wells et al., 2014).

With a rare incidence and their common location in the myometrium especially LMS, an accurate preoperative diagnosis is still a challenge. Certain number of patients undergo surgery for an indication of benign tumors (Ulrich 2015). When a correct diagnosis is made preoperation, tumor stage is assigned by surgical staging comprising of extrafascial hysterectomy, salpingooophorectomy and lymph node resection, which is also the mainstay of treatment (Prat, 2009). Slightly different from its carcinoma counterpart, ovarian preservation and exemption of lymph node resection are sometimes acceptable particular in younger age patients and in those with certain types of sarcoma (Leitao et al., 2003; Kapp et al., 2008). Adjuvant therapy after surgery has evolved over the years without a clear benefit on survival (Cearbhail and Hensley, 2010; Rauh-Hain and Carmen, 2013; Cantrell et al., 2015).

Despite being derived from various cellular origins and having different pathologic features, most sarcomas have aggressive behavior, high rates of recurrence and poor prognosis even when diagnosed at an early stage. Their prognoses remained unchanged: overall 5-year survival ranged from $18 \%$ to $55 \%$ (Kelly and Craighead, 2005; Koivisto et al., 2008; Park et al., 2008; Durnali et al., 2012). Being rare tumors, large prospective studies or randomized controlled trials of uterine sarcoma are 
not common. Availability of more number of reports is important to serve as pieces of jigsaw to build up more data. The aim of this study was to determine clinical presentation, surgical practices, adjuvant therapy, survival outcomes and prognostic factors of uterine sarcomas treated in our institution.

\section{Materials and Methods}

Our study obtained an approval from the Ethical Committees of the institution. Patients diagnosed with uterine sarcomas were identified from the archives of Gynecologic Oncology unit. Inclusion criteria were: patients with uterine sarcoma who had primary surgery in the institution between January 1994 and December 2014. Patients who had operation in other hospitals who were referred for further treatment were also included. Uterine sarcoma in this study included: pure sarcoma of LMS, ESS, UUS; mixed epithelial and mesenchymal tumors of AS and CS. Exclusion criteria were: patients with soft tissue sarcoma or sarcoma from other gynecologic organs which metastasized to the uterus and patients who had primary treatment elsewhere and did not have pathological slides for review, or pathological reports upon referral. The patients who had incomplete data were also excluded.

As a general practice in the institution, surgery was performed by general gynecologist if diagnosis was benign disease, or gynecologic oncologist if pre-operative tissue diagnosis or clinical suspicion for malignant disease. A decision of adjuvant therapy was made in a multidisciplinary clinic among gynecologic oncologists, radiation oncologists, with or without medical oncologists based on surgical findings, pathology, and performance status of the patients as well as their acceptance.

Data collected were: age at diagnosis, menopausal status, surgical procedures, residual disease, stage, pathological data of tumors, adjuvant therapy, status of disease, and survival. Pathological data included histopathological type, tumor size, depth of myometrial invasion, and lymphovascular space invasion ( $\mathrm{L} V s \mathrm{I})$. Stage of all patients including those who were diagnosed prior to 2009 were assigned or re-assigned according to the 2009 International Federation of Gynecology and Obstetrics (FIGO). Progression-free survival (PFS) was calculated from date of diagnosis until date of progression, recurrence, or death. Overall survival (OS) was obtained from date of diagnosis to date of death. For the patients who were alive, data were censored on the date of last follow-up visit.

Statistical analysis was performed using SPSS 22.0 for windows (IBM Corporation, Armonk, NY, USA). Descriptive statistics were used to analyze demographic data and were summarized as numbers with percentage, mean with standard deviation or median with range. Data were compared by Student t test or Wilcoxon-MannWhitney test and Chi Square and Fisher's exact test as

Table 1. Clinical Features and Primary Treatment of Patients with Uterine Sarcoma (N=46)

\begin{tabular}{|c|c|c|c|c|}
\hline General characteristic features & All sarcoma & Carcinosarcoma & Leiomyosarcoma & Stromal sarcoma \\
\hline & $\mathrm{n}=46(\%)$ & $\mathrm{n}=25(\%)$ & $\mathrm{n}=15(\%)$ & $\mathrm{n}=6(\%)$ \\
\hline Age, mean $\pm \mathrm{SD}$ & $54.0 \pm 11.9$ & $60.3 \pm 9.2$ & $49.3 \pm 10.1$ & $39.8 \pm 9.28$ \\
\hline \multicolumn{5}{|l|}{ Menopause status } \\
\hline Premenopause & $12(26.1)$ & - & $7(46.7)$ & $5(83.3)$ \\
\hline Postmenopause & $34(73.9)$ & $25(100.0)$ & $8(53.3)$ & $1(16.7)$ \\
\hline \multicolumn{5}{|l|}{ Parity } \\
\hline 0 & $26(56.5)$ & $13(52.0)$ & $8(53.3)$ & $5(83.3)$ \\
\hline$\geq 1$ & $20(43.5)$ & $12(48.0)$ & $7(46.7)$ & $1(16.7)$ \\
\hline \multicolumn{5}{|l|}{ Presenting symptoms } \\
\hline Abnormal vaginal bleeding & $29(63.0)$ & $23(92.0)$ & $2(13.3)$ & $4(66.7)$ \\
\hline Mass with or without bleeding & $17(37.0)$ & $2(8.0)$ & $13(86.7)$ & $2(33.3)$ \\
\hline \multicolumn{5}{|l|}{ Preoperative Biopsy } \\
\hline Not done & $13(28.3)$ & $1(4.0)$ & $10(66.7)$ & $2(33.3)$ \\
\hline Done & $33(71.7)$ & $24(96.0)$ & $5(33.3)$ & $4(66.7)$ \\
\hline Correct diagnosis & $29\left(87.9^{*}\right)$ & $24(100.0 *)$ & $2\left(40.0^{*}\right)$ & $3(75.0 *)$ \\
\hline \multicolumn{5}{|l|}{ Primary surgeon } \\
\hline General gynecologist & $9(19.6)$ & - & $8(53.3)$ & $1(16.7)$ \\
\hline Gynecologic oncologist & $37(80.4)$ & $25(100.0)$ & $7(46.7)$ & $5(83.3)$ \\
\hline \multicolumn{5}{|l|}{ Type of surgery } \\
\hline Hysterectomy & $3(6.5)$ & - & $1(6.7)$ & $2(33.3)$ \\
\hline Hysterectomy with BSO & $43(93.5)$ & $25(100.0)$ & $14(93.3)$ & $4(66.7)$ \\
\hline \multicolumn{5}{|l|}{ Lymph node resection } \\
\hline Yes & $34(73.9)$ & $25(100.0)$ & $5(33.3)$ & $4(66.7)^{* *}$ \\
\hline No & $12(26.1)$ & - & $10(66.7)$ & $2(33.3)$ \\
\hline \multicolumn{5}{|l|}{ Outcome of surgery } \\
\hline No residual tumor & $41(89.1)$ & $20(80.0)$ & $15(100.0)$ & $6(100.0)$ \\
\hline Residual tumor & $5(10.9)$ & $5(20.0)$ & - & - \\
\hline
\end{tabular}

Stromal sarcoma include 6 patients ( 2 endometrial stromal sarcoma $\{\mathrm{ESS}\}, 2$ adenosarcoma $\{$ AS $\}$ and 2 undifferentiated uterine sarcoma $\{$ UUS $\}$ );

* Percentage of correct diagnosis was obtained from the number of patients who had pre-operative tissue dianosis; ** One patient with UUS had only pelvic node resection while others had both pelvic and para-aortic node resection 
appropriate. Survivals were analyzed by the KaplanMeier method and were compared by log-rank test and Cox regression analysis. Any p-values of $<0.05$ were considered significant.

\section{Results}

Clinical characteristics of uterine sarcoma patients

During the study period, 53 patients were identified from the archives of the institution. Seven were excluded for the following reasons: 3 had sarcoma of non-uterine origin, 2 had CS from uterine curettage who were referred to other hospitals for management due to reimbursement policy, and 2 had no available clinical and final pathologic data. Total of 46 patients met inclusion criteria and were included in the study. Histologic subtypes in order of frequency were: CS 25 cases $(54.3 \%)$; LMS 15 cases (32.6\%); and 2 cases each of UUS, ESS, or AS (13.1\%).
Mean age of all patients was 54.0 \pm 11.9 years (range 25-82 years). Patients with CS were older than the other groups, 60.3 years old compared to 49.3 years of LMS and 39.8 years of other sarcomas (p-value 0.001 and $<0.001$ respectively). Thirty-four patients (74\%) were menopausal: all in CS, approximately half in LMS, and only one patient with stromal sarcomas who aged 49 years.

Abnormal uterine bleeding was the most common presenting symptoms being found in 29 patients $(63.0 \%)$ followed by pelvic or abdominal mass with or without bleeding in the other 17 patients $(37.0 \%)$. Almost all patients with CS (23 patients or $92 \%$ ) presented with abnormal uterine bleeding. On the other hand, 13/15 of LMS $(86.7 \%$ ) had main symptom of pelvic or abdominal mass.

Out of 46 patients, 33 (71.7\%) had endometrial biopsy or uterine curettage for tissue diagnosis pre-operation due to abnormal bleeding ( 29 patients) or pelvic mass with

Table 2. Surgico-Pathologic Features of Uterine Sarcoma $(\mathbf{N}=46)$

\begin{tabular}{|c|c|c|c|c|}
\hline & All sarcoma & Carcinosarcoma & Leiomyosarcoma & Stromal sarcoma \\
\hline & $\mathrm{n}=46(\%)$ & $\mathrm{n}=25(\%)$ & $\mathrm{n}=15(\%)$ & $\mathrm{n}=6(\%)$ \\
\hline Median size, $\mathrm{cm}$ (range) & $6.0(2-16)$ & $6.0(3-15)$ & $9.0(3-16)$ & $4.5(2-6)$ \\
\hline \multicolumn{5}{|l|}{ FIGO Stage } \\
\hline I & $26(56.5)$ & $8(32.0)$ & $13(86.7)$ & $5(83.3)$ \\
\hline II & $1(2.2)$ & - & - & $1(16.7)$ \\
\hline III & $13(28.3)$ & $11(44.0)$ & $2(13.3)$ & - \\
\hline IV & $6(13.0)$ & $6(24.0)$ & - & - \\
\hline \multicolumn{5}{|l|}{$\operatorname{LVSI}(n=37)$} \\
\hline No & $15(40.5)$ & $6(26.1)$ & $6(66.7)$ & $3(60.0)$ \\
\hline Yes & $22(59.5)$ & $17(73.9)$ & $3(33.3)$ & $2(40.0)$ \\
\hline \multicolumn{5}{|l|}{ Myometrial invasion } \\
\hline$<50$ & $12(26.1)$ & $4(16.0)$ & $3(20.0)$ & $5(83.3)$ \\
\hline$>50 \%$ & $34(73.9)$ & $21(84.0)$ & $12(80.0)$ & $1(16.7)$ \\
\hline \multicolumn{5}{|l|}{ Nodal metastasis } \\
\hline PN metastasis/ N desected & 6/34 (17.6) & $6 / 25(24.0)$ & - & - \\
\hline $\mathrm{PN}$ and $\mathrm{PAN} *$ & $9 / 33(27.3)$ & $8 / 25(32.0)$ & $1 / 5(20.0)$ & - \\
\hline
\end{tabular}

Stromal sarcoma include 6 patients ( 2 endometrial stromal sarcoma $\{\mathrm{ESS}\}, 2$ adenosarcoma $\{$ AS $\}$ and 2 undifferentiated uterine sarcoma $\{\mathrm{UUS}$ ); Abbreviations: FIGO, International Fenderation of Obstetrics; LVSI, lymphovascular invasion; PN, pelvic nodes; PAN, para-aortic nodes; N, noodes * All patients with para-aortic node metastasis also had pelvic node metastasis

Table 3. Adjuvant Therapy and Outcomes of Patients with Uterine Sarcoma (N=46)

\begin{tabular}{|c|c|c|c|c|}
\hline Type of & All sarcoma & Carcinosarcoma & Leiomyosarcoma & Stromal sarcoma \\
\hline & $\mathrm{n}=46(\%)$ & $\mathrm{n}=25(\%)$ & $\mathrm{n}=15(\%)$ & $\mathrm{n}=6(\%)$ \\
\hline \multicolumn{5}{|l|}{ Adjuvant therapy } \\
\hline No & $19(41.3)$ & $9(36.0)$ & $6(40.0)$ & $4(66.7)$ \\
\hline Yes & $27(58.7)$ & $16(64.0)$ & $9(60.0)$ & $2(33.3)$ \\
\hline \multicolumn{5}{|l|}{ Type of adjuvant therapy } \\
\hline Radiation & $7(15.2)$ & $5(20.0)$ & $2(13.3)$ & - \\
\hline Chemotherapy & $12(26.1)$ & $4(16.0)$ & $7(46.7)$ & $1(16.7)$ \\
\hline Chemotherapy\& Radiation & $7(15.2)$ & $7(28.0)$ & - & - \\
\hline Hormonal Therapy & $1(2.2)$ & - & - & $1(16.7)$ \\
\hline \multicolumn{5}{|l|}{ Treatment outcomes } \\
\hline No evidence of disease & $19(41.3)$ & $8(32.0)$ & $8(53.3)$ & $3(50.0)$ \\
\hline Progression & $5(10.9)$ & $5(20.0)$ & - & - \\
\hline Recurrence & $22(47.8)$ & $12(48.0)$ & $7(46.7)$ & $3(50.0)$ \\
\hline \multicolumn{5}{|l|}{ Site of progression/ recurrence } \\
\hline Local & $4(9.8)$ & $4(23.5)$ & $1(6.7)$ & - \\
\hline Distant & $11(40.7)$ & $6(35.3)$ & $5(71.4)$ & - \\
\hline Local and distant & $11(40.7)$ & $7(41.2)$ & $1(14.3)$ & $3(100.0)$ \\
\hline
\end{tabular}

Stromal sarcoma include 6 patients ( 2 endometrial stromal sarcoma $\{\mathrm{ESS}\}, 2$ adenosarcoma $\{$ AS $\}$ and 2 undifferentiated uterine sarcoma $\{$ UUS $\}$ ) 


\begin{tabular}{lcccc}
\hline \multirow{2}{*}{ Adjuvant treatment } & \multicolumn{4}{c}{ Disease outcome (n of each histopathology) } \\
\cline { 2 - 5 } & No recurrence & LR & DR & LR and DR \\
\hline No adjuvant Rx $(\mathrm{n}=19)$ & $7(2 \mathrm{CS}, 4 \mathrm{LMS}, 1 \mathrm{SS})$ & $4(3 \mathrm{CS}, 1 \mathrm{LMS})$ & 1 (LMS) & 7 (4 CS, 3 SS) \\
Adjuvant Rx $(\mathrm{n}=27)$ & 12 & 1 & 10 & 4 \\
RT $(\mathrm{n}=7)$ & $4(3 \mathrm{CS}, 1 \mathrm{LMS})$ & - & $3(2 \mathrm{CS}, 1 \mathrm{LMS})$ & - \\
CMT $(\mathrm{n}=12)$ & $6(2 \mathrm{CS}, 3 \mathrm{LMS}, 1 \mathrm{SS})$ & - & $4(1 \mathrm{CS}, 3 \mathrm{LMS})$ & $2(1 \mathrm{CS}, 1 \mathrm{LMS})$ \\
RT + CMT $(\mathrm{n}=7)$ & 1 (CS) & 1 (CS) & $3(3 \mathrm{CS})$ & $2(2 \mathrm{CS})$ \\
HT $(\mathrm{n}=1)$ & 1 (SS) & - & - & - \\
Total $(\mathrm{N}=46)$ & 19 & 5 & 11 & 11 \\
\hline
\end{tabular}

CMT, chemotherapy; CS, carcinosarcoma; DR, distant recurrence; HT, hormonal therapy; LMS, leiomyosarcoma; LR, local recurrence; SS, stromal sarcoma; Rx, treatment

Table 5. Univariate Analysis for Progression-Fee Survival and Overall Survival

\begin{tabular}{|c|c|c|c|c|c|c|}
\hline & \multirow{2}{*}{$\frac{2-y r \text { PFS }}{(95 \% \mathrm{CI})}$} & \multirow{2}{*}{ P-value } & HR & \multirow{2}{*}{$\frac{2-y r \text { OS }}{(95 \% \mathrm{CI})}$} & \multirow{2}{*}{ P- value } & \multirow{2}{*}{$\begin{array}{c}\mathrm{HR} \\
(95 \% \mathrm{CI}) \\
\end{array}$} \\
\hline & & & $(95 \% \mathrm{CI})$ & & & \\
\hline \multicolumn{7}{|l|}{ Age } \\
\hline$\leq 50 \mathrm{yr}(\mathrm{n}=15)$ & $65.5(40.8-90.1)$ & 0.034 & 1 & $64.3(39.2-89.4)$ & 0.056 & 1 \\
\hline$>50$ yr $(n=31)$ & $35.5(18.6-52.3)$ & & $2.59(1.04-6.43)$ & $41.9(24.6-59.3)$ & & $2.37(0.95-5.88)$ \\
\hline \multicolumn{7}{|l|}{ Menopause } \\
\hline No $(n=12)$ & $73.3(47.4-99.3)$ & 0.023 & 1 & 72.7 (46.4-99.1) & 0.032 & 1 \\
\hline Yes $(n=34)$ & $35.3(19.2-51.4)$ & & $3.22(1.11-9.34)$ & $41.2(24.6-57.7)$ & & $3.03(1.04-8.81)$ \\
\hline \multicolumn{7}{|l|}{ LND } \\
\hline Not done $(n=12)$ & $75.0(50.5-99.5)$ & 0.027 & 1 & $83.3(62.2-100.0)$ & 0.026 & 1 \\
\hline Done $(n=34)$ & $34.5(18.3-50.7)$ & & $3.13(1.08-9.07)$ & $36.5(19.9-53.0)$ & & $3.17(1.09-9.23)$ \\
\hline \multicolumn{7}{|l|}{ Outcome of surgery } \\
\hline $\begin{array}{l}\text { No residual tumor } \\
(\mathrm{n}=41)\end{array}$ & $50.7(35.2-66.1)$ & $<0.001$ & 1 & $52.6(37.1-68.1)$ & $<0.001$ & 1 \\
\hline Residual tumor $(\mathrm{n}=5)$ & - & & $5.64(2.70-11.81)$ & $20.0(0-55.1)$ & & $2.31(1.40-3.81)$ \\
\hline \multicolumn{7}{|l|}{ FIGO Stage } \\
\hline Early (I-II) (n=27) & $66.1(48.1-84.2)$ & $<0.001$ & 1 & $69.3(51.6-87.0)$ & $<0.001$ & 1 \\
\hline $\begin{array}{l}\text { Advanced (III-IV) } \\
(n=19)\end{array}$ & $15.8(0-32.2)$ & & $3.84(1.77-8.35)$ & $21.1(2.7-39.4)$ & & $3.75(1.73-8.16)$ \\
\hline \multicolumn{7}{|l|}{ Adjuvant Treatment } \\
\hline Yes $(n=27)$ & $47.1(27.9-66.2)$ & 0.42 & 1 & $53.8(34.6-73.1)$ & 0.675 & 1 \\
\hline No $(n=19)$ & $42.1(19.9-64.3)$ & & $1.37(0.64-2.93)$ & $42.1(19.9-64.3)$ & & $1.18(0.55-2.53)$ \\
\hline \multicolumn{7}{|l|}{ Adjuvant Chemotherapy } \\
\hline Yes $(n=19)$ & $34.7(12.7-56.8)$ & 0.647 & 1 & $44.7(21.7-67.7)$ & 0.541 & 1 \\
\hline No $(n=27)$ & $51.9(33.0-70.7)$ & & $0.84(0.39-1.80)$ & $51.9(33.0-70.7)$ & & $0.79(0.37-1.69)$ \\
\hline \multicolumn{7}{|l|}{ Adjuvant Radiation } \\
\hline Yes $(n=14)$ & $42.9(16.9-68.8)$ & 0.836 & 1 & $50.0(23.8-76.2)$ & 0.811 & 1 \\
\hline No $(n=32)$ & $46.2(28.7-63.6)$ & & $0.92(0.41-2.05)$ & $48.8(31.2-66.4)$ & & $0.91(0.41-2.03)$ \\
\hline \multicolumn{7}{|l|}{ Histology } \\
\hline $\mathrm{CS}(\mathrm{n}=25)$ & $36.0(17.2-54.8)$ & 0.088 & 1 & $40.0(20.8-59.2)$ & 0.196 & 1 \\
\hline LMS $(n=15)$ & $50.6(24.4-76.7)$ & & $0.47(0.19-1.13)$ & $57.1(31.2-83.1)$ & & $0.51(0.21-1.23)$ \\
\hline Stromal sarcoma $(n=6)$ & $66.7(28.9-100)$ & & $0.49(0.14-1.68)$ & $66.7(28.9-100.0)$ & & $0.49(0.14-1.67)$ \\
\hline \multicolumn{7}{|l|}{ Tumor size } \\
\hline$\leq 5 \mathrm{~cm}(\mathrm{n}=14)$ & $64.3(39.2-89.4)$ & 0.104 & 1 & $64.3(39.2-89.4)$ & 0.091 & 1 \\
\hline$>5 \mathrm{~cm}(\mathrm{n}=32)$ & $36.5(19.6-53.5)$ & & $2.09(0.84-5.20)$ & $42.3(24.9-59.7)$ & & $2.16(0.87-5.38)$ \\
\hline \multicolumn{7}{|l|}{ Myometrial invasion } \\
\hline$\leq 50 \%(n=12)$ & $66.7(40.0-93.3)$ & 0.143 & 1 & $66.7(40.0-93.3)$ & 0.106 & 1 \\
\hline$>50 \%(\mathrm{n}=34)$ & $37.3(20.8-53.7)$ & & $2.05(0.77-5.48)$ & $42.5(25.5-59.4)$ & & $2.25(0.82-6.14)$ \\
\hline \multicolumn{7}{|l|}{ LVSI $(n=37)$} \\
\hline Negative $(n=15)$ & $53.3(28.1-78.6)$ & 0.153 & 1 & $53.3(28.1-78.6)$ & 0.167 & 1 \\
\hline Positive $(n=22)$ & $36.4(16.3-56.5)$ & & $1.91(0.78-4.69)$ & $40.9(20.4-61.5)$ & & $1.87(0.76-4.60)$ \\
\hline
\end{tabular}

HR, Hazard ratio; C.I., Confidence interval; FIGO, International Fenderation of Obstetrics and Gynecology; LND, lymp nodes dissection; CS, carcinosarcoma; LMS, leiomyosarcoma; LVSI, lymphovascular invasion.

bleeding (4 patients). Correct diagnosis of malignancy was made in 29 patients $(87.9 \%)$. However, only 19 patients $(57.6 \%)$ had pre-operative diagnosis of sarcoma. The other
4 patients whose malignancy was not detected were later revealed from hysterectomy to have LMS (3 patients) and ESS (1 patient). 
Table 6. Multivariate Analysis for Progression-Free Survival and Overall Survival

\begin{tabular}{|c|c|c|c|c|}
\hline \multirow{3}{*}{ Variable } & \multicolumn{2}{|c|}{ 2-yr PFS } & \multicolumn{2}{|c|}{$2-y r O S$} \\
\hline & \multirow{2}{*}{$\begin{array}{c}\text { HR } \\
(95 \% \mathrm{CI})\end{array}$} & \multirow{2}{*}{ P-value } & $\mathrm{HR}$ & \multirow{2}{*}{ P-value } \\
\hline & & & $(95 \% \mathrm{CI})$ & \\
\hline \multicolumn{5}{|l|}{ Age } \\
\hline$\leq 50 \mathrm{yr}(\mathrm{n}=15)$ & 1 & \multirow[t]{2}{*}{0.558} & 1 & \multirow{2}{*}{0.784} \\
\hline$>50$ yr $(n=31)$ & $1.58(0.35-7.21)$ & & $1.23(0.27-5.57)$ & \\
\hline \multicolumn{5}{|l|}{ Menopause } \\
\hline No $(n=12)$ & 1 & \multirow[t]{2}{*}{0.84} & 1 & \multirow{2}{*}{0.729} \\
\hline Yes $(n=34)$ & $1.21(0.19-7.58)$ & & $1.38(0.22-8.67)$ & \\
\hline \multicolumn{5}{|l|}{ LND } \\
\hline Not done $(n=12)$ & 1 & \multirow[t]{2}{*}{0.357} & 1 & \multirow{2}{*}{0.46} \\
\hline Done $(n=34)$ & $1.72(0.54-5.51)$ & & $1.59(0.47-5.38)$ & \\
\hline \multicolumn{5}{|l|}{ Outcome of surgery } \\
\hline No residual tumor $(n=41)$ & 1 & \multirow[t]{2}{*}{$<0.001$} & 1 & \multirow{2}{*}{0.113} \\
\hline Residual tumor $(\mathrm{n}=5)$ & $15.31(3.31-70.75)$ & & $2.46(0.81-7.45)$ & \\
\hline \multicolumn{5}{|l|}{ FIGO Stage } \\
\hline $\operatorname{Early}(\mathrm{I}, \mathrm{II})(\mathrm{n}=27)$ & 1 & \multirow[t]{2}{*}{0.083} & 1 & \multirow{2}{*}{0.091} \\
\hline Advanced(III,IV) $(n=19)$ & $2.27(0.90-5.73)$ & & $2.27(0.88-5.85)$ & \\
\hline
\end{tabular}

HR, Hazard ratio; C.I., Confidence interval; FIGO, International Fenderation of Obstetrics and Gynecology; LND, lymp nodes dissection.

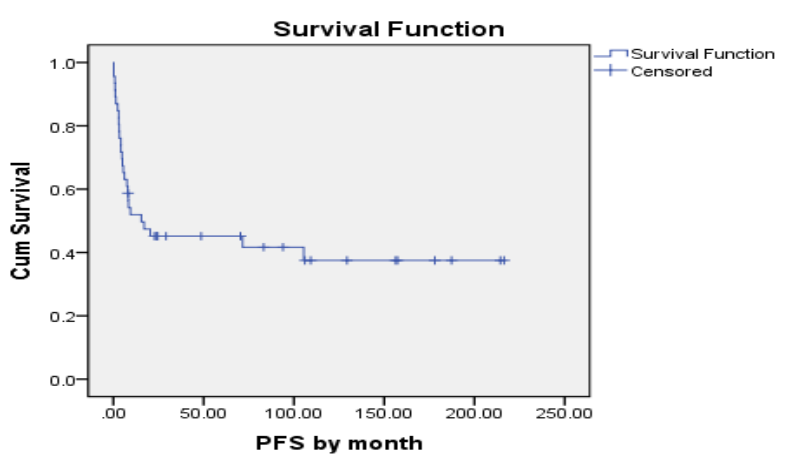

Figure 1. Two-year Progression-Free Survival

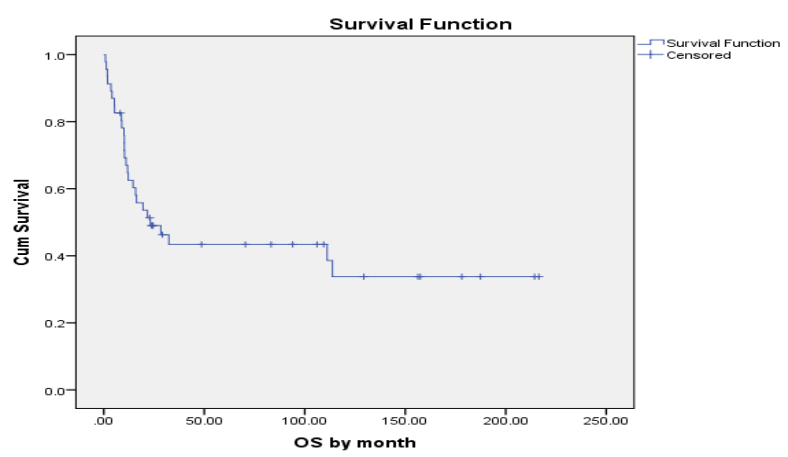

Figure 2. Two-year Overall Survival

All of our uterine sarcoma patients had primary surgical treatment. The operation was performed by gynecologic oncologists in 37 patients $(80.4 \%)$. Simple hysterectomy was performed in 44 patients $(95.7 \%)$ while the other 2 had radical hysterectomy for gross cervical involvement. The ovaries were preserved in 3 patients: 2 UUS patients who aged 29 or 30 years and 1 LMS who aged 25 years. Retroperitoneal lymph nodes were resected in 34 patients (73.9\%): all patients with CS and only 9/21 patients $(42.9 \%)$ with other sarcomas. Outcome of the surgery was optimal with no residual tumors in 41 patients $(89.1 \%)$ and suboptimal with gross residual tumors in

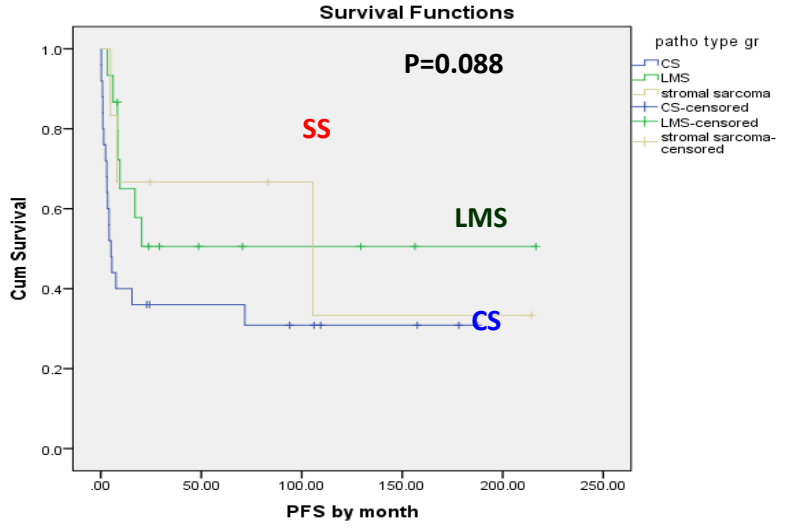

Figure 3. Two-year PFS by Histologic Subtype

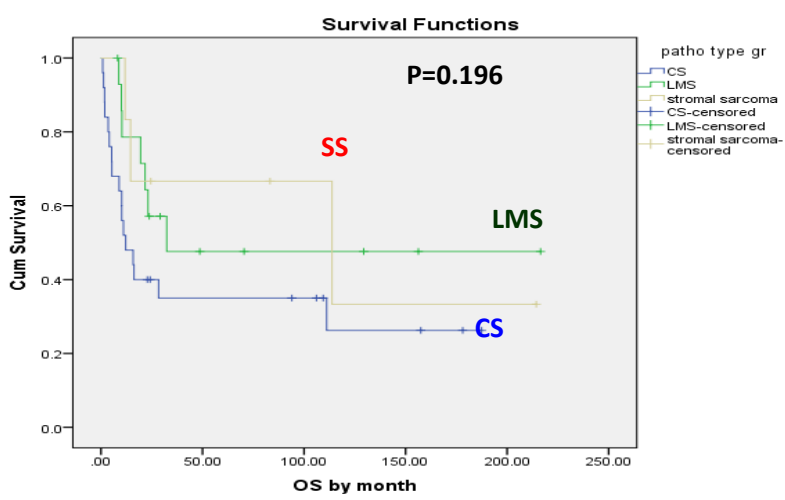

Figure 4. Two-year OS by Histologic Subtype

5 (all in CS group). Clinical characteristic features and primary treatment of the patients are shown in Table 1.

Regarding pathologic features, median size of primary tumor was $6.0 \mathrm{~cm}$ (range $2-16 \mathrm{~cm}$ ). Leiomyosarcoma was the largest among all, with median size of $9.0 \mathrm{~cm}$ compared to $6.0 \mathrm{~cm}$ of CS ( $\mathrm{p}=0.028)$ and $4.5 \mathrm{~cm}$ of stromal sarcomas $(\mathrm{p}=0.007)$. Majority or 34 patients $(73.9 \%)$ had more than half of myometrial invasion. Among 37 patients who had pathological notes for the presence of 
LVsI, 22 (59.5\%) had LVsI. Among 34 patients who had retroperitoneal nodal resection, metastases were evident in 15 patients $(45.5 \%)$, and being most common in CS (14 patients or $93.3 \%$ ). The most common stage for all uterine sarcoma patients was stage I (26 patients or $56.5 \%$ ) followed by stage III (13 patients or $28.3 \%$ ) and stage IV (6 patients or $13.0 \%$ ) while stage II was least frequently found (1 patients or 2.2\%). Type and outcome of surgery, and surgico-pathologic features of the tumors are shown in Table 2.

From 46 patients, adjuvant treatment was given to 27 patients (58.7\%): 16 with early stage $(59.3 \%$ of early stage group) and 11 with advanced stage (57.9\% of advanced stage) .The most common type of adjuvant treatment was chemotherapy, given to 19 patients $(41.3 \%$ of all or $70.4 \%$ among those who had adjuvant treatment). Chemotherapy regimens varied from doxorubicin (1 patient), doxorubicin with ifosfamide (4 patients), cisplatin with ifosfamide (1 patient) or with doxorubicin (2 patients), paclitaxel with carboplatin (7 patients) and no data of regimen (4 patients). Radiation alone was used in 7 patients (15.2\%), while hormonal treatment was given to only one patient who had ESS. Among 19 patients who did not receive any adjuvant treatment, 11 had early stage disease without any risk factors while the other 8 patients who were in advanced stage did not have adjuvant treatment because they had poor performance status after surgery $(n=5)$ or declined $(n=3)$.

Survival of uterine sarcoma patients and prognostic factors

After a median follow-up of 16.0 months (range 0.8187.4 months), 5 patients $(10.9 \%)$ had rapid progression of diseases (median time 0.9 months, range 0.2-3.0 months). All had stage IV CS (only one of them was being treated with chemotherapy after radiation). Recurrences were encountered in 22 patients $(47.8 \%)$. Median time to recur was 5.8 months (range 1.0-105.5 months). The one ESS patient with stage I disease had long disease-free interval of 105.5 months without adjuvant treatment. They were in stage I ( $\mathrm{n}=11: 3$ CS , 5 LMS , 3 stromal sarcomas), stage III ( $n=10: 8$ CS, 2 LMS), and stage IV ( $n=1$ : CS with omental metastasis with no residual disease after surgery). Among these recurrences, 14 of them (63.6\%) received adjuvant therapy (6 in stage I, 7 stage 3 , one stage IV). Regarding the site of recurrence, distant failure was more common (22 patients or $81.4 \%$ ), being isolated or combined locoregional/ distant in equal numbers (11 each). The other recurrences remained loco-regional (5 patients or 18.6\%). The most common site of distant failure was pulmonary metastases. Table 3 shows type of adjuvant therapy and disease outcomes after treatment. Number of patients according to the sites of recurrences by type of adjuvant treatment is demonstrated in Table 4.

Median PFS was 12.5 months (range 0.2-216.5 months) with 2-year PFS of $45.2 \%$ (95\% confidence interval $[\mathrm{CI}], 30.6 \%-59.7 \%)$. Among 27 progression/ recurrences, $7(25.9 \%)$ were subsequently treated with chemotherapy, $2(7.4 \%)$ with combined chemotherapy and radiation, and $18(66.7 \%)$ had only palliative treatment to relieve their symptoms. Median time from recurrence to death in 9 patients who had further treatment was significantly longer than 18 patients who had only palliative treatment: 9.2 months (range 2.7-39.4 months) vs 2.1 months (range 0.1-16.2 months) ( $\mathrm{p}=0.003$ ). All patients who had progression/ recurrences including the patient who had late recurrence died of their diseases. At the time of this report, 16 including 2 patients who had ovarian preservation $(34.8 \%)$ were alive without any evidence of disease. Median overall survival was 22.3 months (range 0.8-216.5 months) with 2-year OS of $48.3 \%$ (95\% CI, 33.3\%-60.7\%).

We studied prognostic factors and found that menopause, lymphadenectomy, residual tumor after surgery and stage were significantly associated with PFS and OS. Table 5 shows univariable analyses of PFS and OS according to prognostic factors. Entering these significant factors into multivariable analyses, we found that residual disease after surgery was the only significant factor for PFS and none for OS (Table 6).

\section{Discussion}

Although CS has recently been regarded as metaplastic carcinoma based on basic ultra-structure (McCluggage 2002). It is still classified by the World Health Organization as a mixed epithelial and mesenchymal tumor base on histomorphologic appearance (Wells et al., 2014). Our previous works had included data of CS patients in the studies of endometrial carcinoma addressing the issues of adjuvant therapy (Tangjitgamol et al., 2015), medical morbidities (Tangjitgamol et al., 2014) or other cancers (unpublished observations), however, these studies did not focus outcomes by histologic subtypes. Base on the WHO classification and by the fact that most studies compared different clinical features of CS with other uterine sarcomas, we identified more number of CS in recent years and compared it with other sarcomas in this study.

Our study found CS as the most common type of uterine sarcoma (approximately one half), followed by LMS (nearly one third). Our data were consistent with Benito et al. study which found CS in $48 \%$ and LMS in $22 \%$ (Benito et al., 2009). Contrast with other studies in Thailand, most common type are LMS .Other previous studies reported different findings, with similar frequency of CS and LMS (Kelly and Craighead, 2005; Koivisto et al., 2008; Park et al., 2008; Durnali et al., 2012). The small number of cases in each study might have exaggerated the different proportion of each uterine sarcoma type. Nevertheless, our study and all other previous studies found ESS or AS as the least common constituting only 10-30\% (Kelly and Craighead, 2005; Koivisto et al., 2008; Park et al., 2008; Benito et al., 2009; Durnali et al., 2012). Our patients' mean age of 54 years was within the range of 50 to 62 years reported in other studies (Livi et al., 2003; Park et al., 2008).

Symptoms of the patients with uterine sarcoma may vary depending on the type or location of sarcoma. Previous studies reported $59-82 \%$ of patients had abnormal uterine bleeding and $17-23 \%$ presented with mass (Kelly and Craighead, 2005; Park et al., 2008; Benito et al., 2009). These presenting symptoms and appropriate 
pre-operative investigation should lead to a correct preoperative diagnosis. Our sarcoma patients had uterine bleeding as the most common symptom, probably due to a high percentage of CS in our series. With the same token, these resulted in high $88 \%$ correct pre-operative diagnoses of malignancy. Others also reported high accuracy of $63 \%$ to $86 \%$ of pre-operative tissue diagnosis of malignancy especially for CS (Bansal et al., 2008; Benito et al., 2009): $90 \%$ for CS and $76 \%$ for other sarcomas (Bansal et al., 2008).

Hysterectomy and bilateral salpingo-oophorectomy was the main treatment for all types of uterine sarcomas. In pre-menopausal patients, ovaries may be preserved especially in early stage LMS and stromal sarcoma because of a low risk of metastases and without an impact on survival (Leitao et al., 2003; Kapp et al., 2008). Only 3 young age or nulliparous patients in our study (6.5\%) had ovarian preservation with different prognosis. Two of them (LMS or UUS) were still alive without any evidence of disease at the time of this report. Another one with UUS was dead 8 month after surgery from pelvic and lung metastases. Hence, we could not make any recommendation for ovarian preservation from this limited number of patients. Surgical evaluation of lymph node was included in the FIGO staging procedures of uterine sarcomas (Prat, 2009). However, lymph node resection is not strictly required for LMS because many studies found less than 5\% nodal involvement in LMS, and the procedure exemption had no impact on survival (Leitao et al., 2003; Kapp et al., 2008). Regarding stage of disease, nearly $60 \%$ of our patients had early stage. This was comparatively low compared to other studies which reported 60\%-76\% ( Koivisto et al., 2008; Park et al., 2008; Benito et al., 2009; Durnali et al., 2012). The differences might lie on the extent of operative procedure especially lymph node surgical evaluation and the proportion of the type of sarcoma. Our institution is a tertiary center for cancer care and most of the surgical procedures were performed by the gynecologic oncologist who generally performed complete surgical staging if the diagnosis of malignancy was made pre- or intra-operation. As found in our study that approximately $74 \%$ of our patients had lymph node resection (all CS and only $43 \%$ of other sarcoma) and majority of CS patients had advanced stage diseases (90\%). With the same reason, the majority of our patients had operation done by the gynecologic oncologists; this may result in a high rate of $89 \%$ having no gross residual tumors. This surgical outcome certainly influenced the use and type of adjuvant therapy aside from stage of disease and the type of tumor.

Regarding adjuvant therapy, the only one randomized trial of adjuvant radiation therapy found limited benefit of improved local control in early stage CS but without benefit on survival (O'Cearbhail and Hensley, 2010). Adjuvant chemotherapy was also studied in an attempt to reduce distant failure and improve survival. Previous reports studied cisplatin, Ifosfamide or doxorubicin for all types of sarcoma, with response rates of 19-36\% (Muss et al., 1985; Thigpen et al., 1991; Kushner et al., 2000). More recent studies, focusing on CS, demonstrated benefit of ifosfamide with cisplatin (Sutton et al., 2000) or with paclitaxel (Homesley et al., 2007) over ifosfamide alone in terms of higher response rates and survivals. Other study showed paclitaxel with carboplatin could yield response rate of 54\% with tolerated toxicity (Powell et al., 2010). For LMS, studies in either soft tissue or uterine sarcoma found high response rates of 16-53\% from docetaxel and gemcitabine (Maki et al., 2007; Hensley et al., 2009). For ESS, hormonal therapy has a certain role (Rauh-Hain and Carmen, 2013).

Adjuvant treatment was almost equally given to early or advanced stage in our study, 59\% and 58\%. This was mainly because our advanced stage patients deteriorated rapidly after surgery that further treatment was not possible. Regarding the type of adjuvant treatment, our study commonly used chemotherapy with or without radiation (70\% of those who had adjuvant treatment) while radiation was used in only $15 \%$. Benefit on local control from RT was demonstrated for both CS and LMS (Table 4). However, the number of patients was too small to draw any conclusion. Our patients had various regimens of chemotherapy due to a long period of treatment when new data of chemotherapy has emerged. The number of patients was too small to evaluate the results by type of adjuvant radiation or chemotherapy regimen. We were able to point out the overall treatment outcomes by stage and type of adjuvant treatment (Table 4). Furthermore in clinical practice, the use of adjuvant therapy did not base solely on histological type, stage, or status of disease post-operation but also on the performance status and the acceptance of the patients and their family (as seen in our 8 advanced stage patients). As seen in our study that our early or advanced stage had similar rate of adjuvant treatment.

Overall prognosis of uterine sarcoma is not good. As demonstrated in our study that $11 \%$ had disease progression within 2-3 months and $48 \%$ had recurrences within a short period of time (median of 6 months) were also experienced even though in early stage. Distant with or without loco-regional failures were the main problem in our series found in approximately $80 \%$ of all recurrences. These were concordant with other studies which found recurrences ranging from $37 \%$ to $63 \%$, being pelvic recurrence in $17-27 \%$, and lung or extraabdominal metastases in 27-55\% (Koivisto et al., 2008; Park et al., 2008; Benito et al., 2009). The patients who had failure after primary treatment had dismal prognosis, despite of salvage treatment except in rare case of ESS. Previous studies showed that, although chemotherapy was used in some patients, $20 \%-30 \%$ had palliative care without specific treatment (Koivisto et al., 2008; Park et al., 2008). This was also found in our study that $63 \%$ of recurrent patients had only palliative care because of poor performance status or denial of further treatment.

The high recurrence rate and ineffective treatment reflected to a close proximity between 2-year PFS and 2-year OS, $45 \%$ and $49 \%$ respectively. Other studies reported 2-year or 3-year OS ranging from 48-64\%(Kelly and Craighead, 2005; Koivisto et al., 2008; Park et al., 2008; Benito et al., 2009; Durnali et al., 2012). A possible reason for different outcomes was various prognostic factors found in each study. These factors 


\section{Chalermrat Potikul et al}

were characteristic features of the patients and their diseases (age, size of tumor, stage of disease, histological types, mitotic figures, grade) as well as the treatment received (type of surgery and surgical outcomes, adjuvant therapy. Our study found significant poor prognostic factors (HR) were: older age (2.59), menopause (3.22), lymphadenectomy (3.13), residual tumor after surgery (5.64), and advanced stage (3.84) (Table 5). Although at different extent, our findings were concordant with previous studies which demonstrated poor prognostic factors as the followings: older age or menopausal patients (Koivisto et al., 2008; Park et al., 2008), suboptimal surgery (Park et al., 2008), advanced stage (Koivisto et al., 2008). In contrast to other reports which did not find any association of lymph node reception and survival (Kelly and Craighead, 2005; Koivisto et al., 2008; Park et al., 2008; Durnali et al., 2012), our study showed significant poor prognosis in patients who had lymph node resection. This might lie on findings that more than half of our patients in this group had advanced stage disease with readily poorer survival. Furthermore, adjuvant therapy was not given as indicated in all of them. Other poor prognostic factors in previous studies of large tumor size (Koivisto et al., 2008; Benito et al 2009), deep myometrium invasion (Park et al., 2008). Particular the histological subtype, endometrial stromal sarcoma was shown to have better prognosis than the others (Maki et al., 2007; Ghaemmaghami et al., 2008). Although we found stromal sarcoma having the best survival among the others, the difference was not significant. This might lie on small number of stromal sarcoma in our series. Furthermore we put ESS together with UUS (with poor prognosis) which might have deteriorated the good prognosis of ESS. Our study had only 2 patients with low-grade ESS, so we did not separately compare them with the other types of sarcoma. For multivariate analysis, we found only residual tumor after surgery was poor prognostic factor for progress free survival. Strength of this study is long term collecting data, but because of uterine sarcoma diagnosis criteria had changed along the time, and this study hadn't review pathologic diagnosis, so it was limitation of this study.

In conclusions, In conclusion, our study elaborated clinical presentation of uterine sarcoma, treatment and outcomes by each histologic subtype. We hope our data should be useful to the general gynecologists and gynecologic oncologists. An awareness of these rare tumors may improve the management and outcomes. High recurrence rate with short survival emphasized a need for future studies to search for an effective adjuvant treatment to reduce recurrence.

\section{Acknowledgements}

This work is granted by medical research fund of Faculty of Medicine Vajira Hospital, Navamindradhiraj University.

\section{References}

Bansal N, Herzog TJ, Burke W, Cohen CJ, Wright JD (2008). The utilty of preoperative endometrial sampling for the detection of uterine sarcomas. Gynecol Oncol, 110, 43-8.

Benito V, Lubrano A, Arencibia O, et al (2009). Clinicopathologic analysis of uterine sarcomas from a single institution in the Canary Islands. Int J Gynaecol Obstet, 1079, 44-9.

Brooks SE, Zhan M, Cote T, Baquet CR (2004). Surveillance, epidemiology and end results analysis of 2677 cases of uterine sarcoma 1989-1999. Gynecol Oncol, 93, 204-8.

Cantrell LA, Blank SV,Duska LR (2015). Uterine carcinosarcoma: A review of the literature. Gynecol Oncol.

Durnali A, Tokluoglu S, Ozdemir N, et al (2012). Prognostic Factors and Treatment Outcomes in 93 Patients with Uterine Sarcoma from 4 Centers in Turkey. Asian Pac J Cancer Prev, 13, 1935-41.

Gao Y, Meng H, Zhang Y, Jiao T, Hui N (2014). Retrospective analysis of 80 cases with uterine carcinosarcoma, leiomyosarcoma and endometrial stromal sarcoma in China,1988-2007. Int J Clin Exp Pathol, 7, 1616-24.

Ghaemmaghami F, Zarchi MK, Gilani MM, et al (2008). Uterine sarcoma : clinicopathological characteristics, treatment and outcomes in Iran. Asian Pac J Cancer Prev, 9, 421-6.

Hensley ML, Ishill N, Soslow R, et al (2009). Adjuvant gemcitabine plus docetaxel for completely resected stages I-IV high grade uterine leiomyosarcoma: Results of a prospective study. Gynecol Oncol, 112, 563-7.

Homesley HD, Filiaci V, Markman M, et al (2007). Phase III trial of ifosfamide with or without paclitaxel in advanced uterine carcinosarcoma: a Gynecologic Oncology Group Study. J Clin Oncol, 25, 526-31.

Kapp DS, Shin JY, Chan JK (2008). Prognostic factors and survival in 1396 patients with uterine leiomyosarcomas: emphasis on impact of lymphadenectomy and oophorectomy. Cancer, 112, 820-30.

Kelly KL, Craighead PS (2005). Characteristics and management of uterine sarcoma patients treated at Tom Baker Cancer Centre. Int J Gynecol Cancer, 15, 132-39.

Koivisto-Korander R, Butzow R, Koivisto A, Leminen A(2008). Clinical outcome and prognostic factors in 100 cases of uterine sarcoma: experience in Helsinki University Central Hospital 1990-2001. Gynecol Oncol, 111, 74-81.

Kushner DM, Webster KD, Belinson JL, et al (2000). Safety and efficacy of adjuvant single-agent ifosfamide in uterine sarcoma. Gynocol Oncol, 78, 221-7.

Leitao MM, Sonoda Y, Brennan MF, Barakat RR, Chi DS (2003). Incidence of lymph node and ovarian metastases in leiomyosarcoma of the uterus. Gynecol Oncol, 91, 209-12.

Livi L, Paiar F, Shah N, et al (2003). Uterine sarcoma: Twentyseven years of experience. Int J Radiation Oncol Biol Phys, 57, 1366-73.

Maki RG, Wathen JK, Patel SR, et al (2007). Randomized phase II study of gemcitabine and docetaxel compared with gemcitabine alone in patients with metastatic soft tissue sarcomas: results of sarcoma alliance for research through collaboration study 002. J Clin Oncol, 25, 2755-63.

McCluggage WG (2002). Uterine carcinosarcomas (malignant mixed Mullerian tumors) are metaplastic carcinomas. Int $J$ Gynecol Cancer, 12, 687-90.

Muss HB, Bundy B, Disaia PJ, et al (1985). Treatment of recurrent or advanced uterine sarcoma. A randomized trial of doxorubicin versus doxorubicin and cyclophosphamide (a phase III trial of the gynecologic oncology group). Cancer, 55, 1648-53.

O'Cearbhail R, Hensley ML (2010). Optimal management of uterine leiomyosarcoma. Expert Rev. Anticancer Ther, 10, 153-69.

Oliva E, Carcangiu ML, Carinelli SG, et al (2014). Mesenchymal tumours. In "WHO classification of tumors of female reproductive organs", Kurman RJ. IARC Press, France 
135-47.

Park JY, Kim DY, Suh DS, et al (2008). Prognostic factors and treatment outcomes of patients with uterine sarcoma: analysis of 127 patients at a single institution, 1989-2007. J Cancer Res Clin Oncol, 134, 1277-87.

Powell MA, Filiaci VL, Rose PG, et al (2010). Phase II evaluation of paclitaxel and carboplatin in the treatment of carcinosarcoma of the uterus: A Gynecologic oncology Group Study. J Clin Oncol, 28, 2727-31.

Prat J (2009). FIGO staging for uterine sarcomas. Int J Gynaecol Obstet, 104, 177-8.

Rauh-Hain JA, del Carmen MG (2013). Endometrial stromal sarcoma: a systematic review. Obstet Gynecol, 122, 676-83.

Reed NS, Mangioni C, Malmström H, et al (2008). Phase III randomized study to evaluate the role of adjuvant pelvic radiotherapy in the treatment of uterine sarcomas stages I and II: an European Organisation for Research and Treatment of Cancer Gynaecological Cancer Group Study (protocol 55874). Eur J Cancer, 44, 808-18.

Sutton G, Brunetto VL, Kilgore L, et al (2000). A phase III trial of ifosfamide with or without cisplatin in carcinosarcoma of the uterus: A Gynecologic Oncology Group Study. Gynecol Oncol, 79, 147-53.

Tangjitgamol S, Khunnarong J, Katanyoo K, et al (2015). Patterns of adjuvant therapy for endometrial cancer: Single institutional experience in Thailand. Int J Gynecol Cancer, 25, 665-72.

Tangjitgamol S, Khunnarong J, Srijaipracharoen S (2014). Medical morbidities in endometrial cancer patients. Int $J$ Gynecol Cancer, 24, 1623-7.

Thigpen JT, Blessing JB, Beecham J, Homesley H, Yordan E (1991). Phase II trial of cisplatin as first-line chemotherapy in patients with advanced or recurrent uterine sarcoma: A Gynecologic Oncology Group study. J Clin Oncol, 9, 1962-6.

Ulrich LG (2015). Benign leiomyoma or malignant sarcoma: The difficult differential diagnosis. Maturitas, 80, 235-6.

Wells M, Oliva E, Palacios J, Prat J (2012). Mixed epithelial and mesenchymal tumours. In "WHO classification of tumors of female reproductive organs", Kurman RJ. IARC Press, France 148-51. 